\title{
Outcome of patients hospitalized by public authorities in a public mental health sector
}

\author{
Françoise Cornic*, Fabien Beghelli and Marie-Noëlle Vacheron
}

\author{
Address: Centre Hospitalier Sainte-Anne, 1 rue Cabanis, 75014 Paris, France
}

* Corresponding author

\author{
from WPA Thematic Conference. Coercive Treatment in Psychiatry: A Comprehensive Review \\ Dresden, Germany. 6-8 June 2007 \\ Published: 19 December 2007 \\ BMC Psychiatry 2007, 7(SuppI I):P4 doi:10.1 I86/I47I-244X-7-SI-P4
}

This abstract is available from: http://www.biomedcentral.com/I47I-244X/7/SI/P4

(c) 2007 Cornic et al; licensee BioMed Central Ltd.

\section{Background}

In France, the laws of 27 June 1990 modified by the law of 4 March 2002 define the scope and procedures of involuntary hospitalizations. Regarding hospitalization by the public authorities the laws state that there must be a threat to the safety of individuals or a serious threat to public order. This procedure is applied since 1838, yet its practice and usefulness have never been assessed. This study seeks to assess current medical practice for applying this procedure and the outcome of the subjects hospitalized under this procedure.

\section{Methods}

A retrospective analysis of clinical data during and after the hospitalization was conducted on 145 subjects hospitalized by the public authorities between 1996 and 2006 in a public mental health sector, in Saint Anna Hospital, Paris, France.

\section{Results}

$90 \%$ of the hospitalization by the public authorities were motivated by an acting out or a high risk of acting out. A majority of patients shared classical characteristics of difficult patients in psychiatric care: men (74\%), socially isolated, with a long psychiatric history (77.8\%), drug addicts (50\%). 25.5\% of the patients had a history of violence and $15 \%$ of them a forensic history. Schizophrenia was the most frequent diagnosis (73\%) according to DSM-IVR criteria. The average hospitalization duration was 2.4 months (2 days to 60 months). After discharge from full-time hospitalization, enforcement of care was maintained using trial discharge for $51 \%$ of the patients.
$9.8 \%$ of the patients were lost to follow-up. During follow-up, $31.4 \%$ were re-hospitalized, only $8 \%$ of them were initially managed in a forensic emergency ward.

\section{Conclusion}

Current medical practice respects hospitalization under the legal frame defined for public authorities. This legal procedure is an efficient tool for psychiatrists. Trial discharge facilitates continuity in psychiatric care and prevents efficiently acting out in difficult patients. 